\title{
Simultaneous occurrence of gastric lipoma and early gastric cancer
}
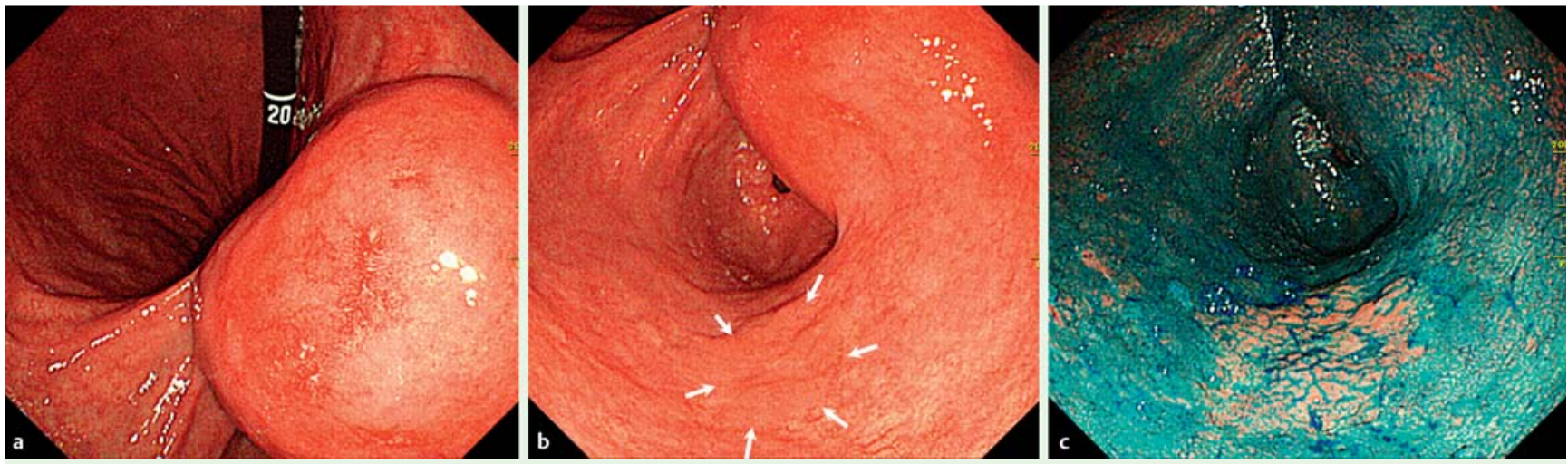

Fig. 1 Endoscopic views showing: a a sessile polypoid lesion with a smooth surface in the greater curvature of the gastric antrum; $\mathbf{b}$ a slightly elevated lesion with a shallow depression (arrows) in the greater curvature of the lower gastric body near the polypoid lesion; $\mathbf{c}$ the distinct borders of a slightly elevated lesion after application of indigo carmine dye.

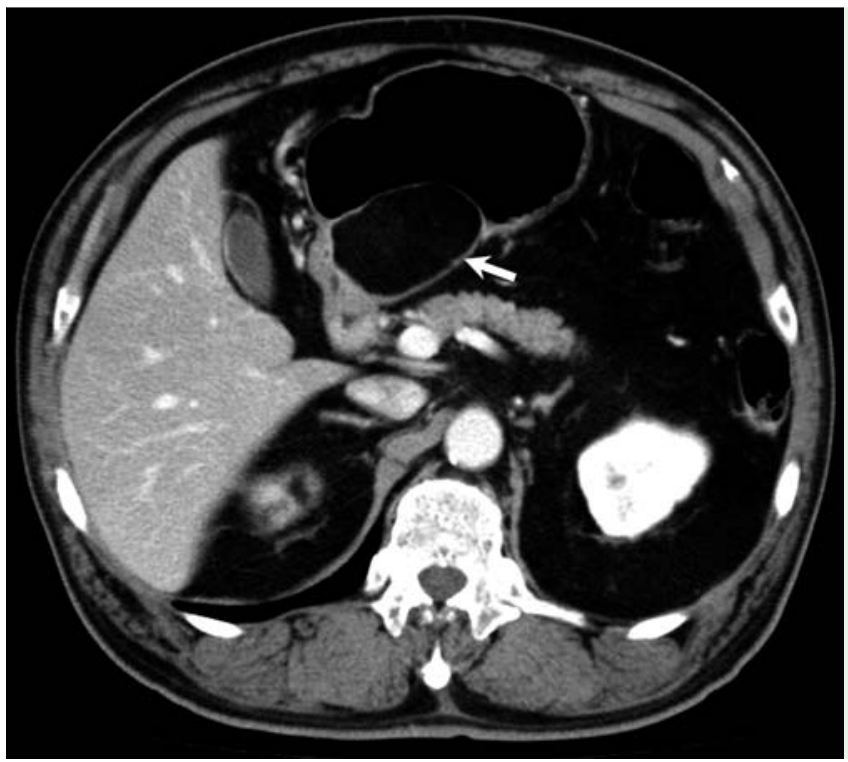

Fig. 2 Abdominal computed tomography (CT) scan showing a well-circumscribed, smooth-bordered, low-density mass in the gastric wall of the antrum (arrow).

An asymptomatic 72-year-old man underwent a screening esophagogastroduodenoscopy (EGD) at our hospital. The EGD showed a sessile polypoid lesion with a smooth surface in the greater curvature of the gastric antrum, which was diagnosed as a submucosal tumor ( $\bullet$ Fig. 1 a). A second slightly elevated lesion with a shallow depression was observed in the greater curvature of the lower gastric body near the polypoid lesion ( $\bullet$ Fig. 1b). This was confirmed on biopsy to be a well-differentiated tubular adenocarcinoma. The borders of this lesion became dis- tinct when indigo carmine dye was used in a chromoendoscopy ( $\bullet$ Fig. 1 c). Abdominal contrast-enhanced computed tomography (CT) revealed a well-circumscribed, low-density mass in the wall of the gastric antrum ( $\bullet$ Fig. 2 ).

The patient underwent endoscopic submucosal dissection (ESD) for the gastric cancer ( Fig.3). Histological examination confirmed the presence of a well-differentiated tubular adenocarcinoma with dimensions of $17 \times 15 \mathrm{~mm}$ that was confined to the gastric mucosal layer without lymphatic and venous infiltration. Subse- quently, partial resection of the stomach was carried out for the submucosal tumor. Gross and histological examination of the resected specimen revealed a well-circumscribed, encapsulated lipoma measuring $8.5 \times 5.5 \mathrm{~cm}$ ( $\mathbf{F i g . 4}$ ). Follow-up after discharge was uneventful. After 8 months, there had been no evidence of metastasis.

The stomach is a rare location for lipomas; only a few cases of gastric lipoma associated with early gastric cancer have been previously reported [1-3]. Interestingly, as in this case, the gastric cancer and the lipoma were also located very close to each other in the three previous cases, suggesting that these were concomitant tumors.

Although lipomas are benign tumors, they can cause gastrointestinal bleeding, intussusception, and obstruction. Therefore minimally invasive therapy with ESD for early gastric cancer and partial gastric resection for large lipomas should be considered. Clinicians should examine patients with gastric tumors carefully, bearing in mind the possibility of synchronous tumors, as demonstrated by this case and the previous cases.

Endoscopy_UCTN_Code_CCL_1AB_2AD_3AB

Competing interests: None 

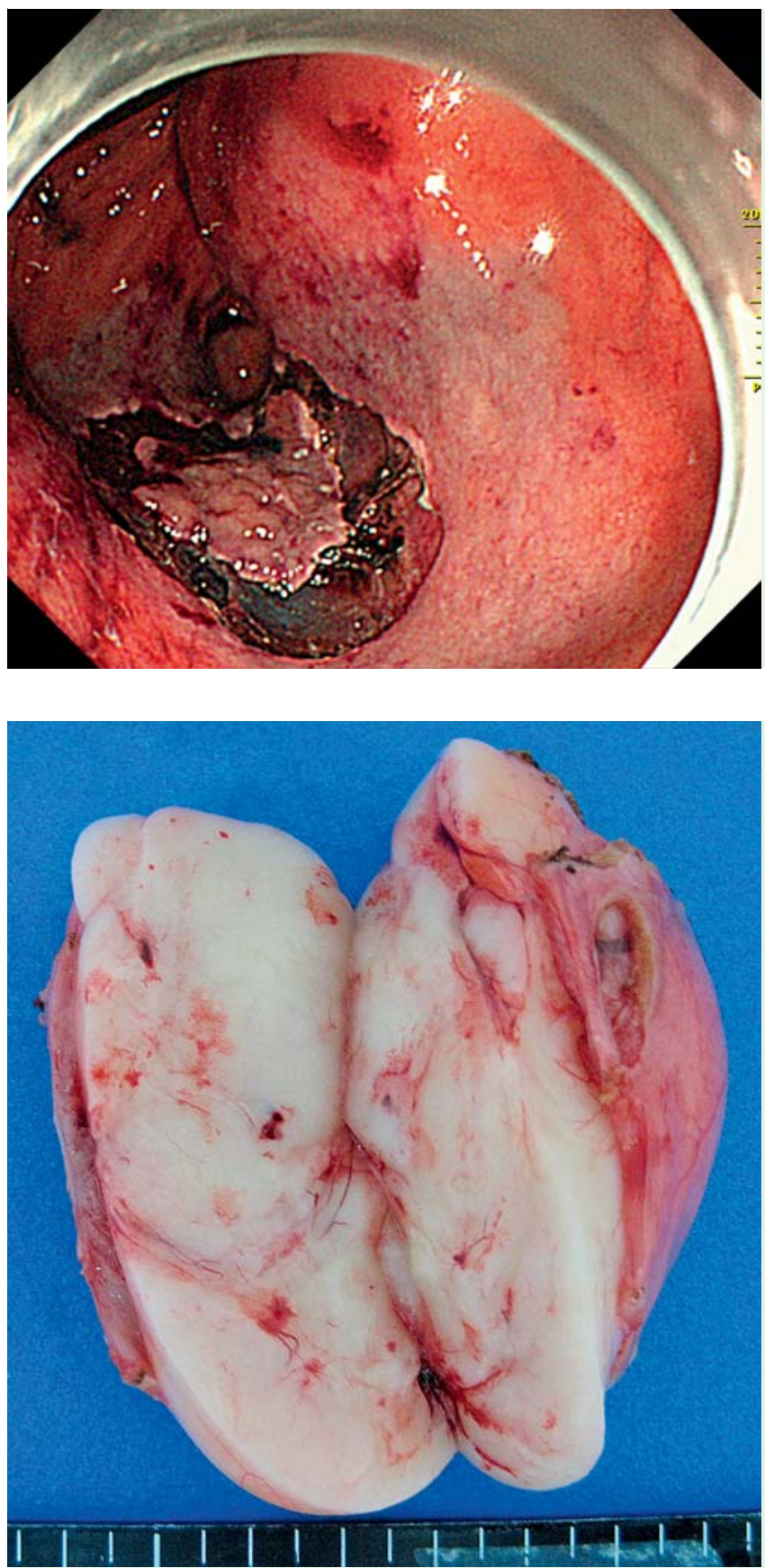

Fig. 3 Endoscopic appearance during endoscopic submucosal dissection (ESD) for the gastric cancer.

Tsutomu Namikawa', Eri Munekage ${ }^{1}$, Hiroshi Mizuta ${ }^{2}$, Michiya Kobayashi ${ }^{1}$, Toshiji Saibara $^{2}$, Kazuhiro Hanazaki ${ }^{1}$

${ }^{1}$ Department of Surgery, Kochi Medical School, Kochi, Japan

${ }^{2}$ Department of Gastroenterology and Hepatology, Kochi Medical School, Kochi, Japan

\section{References}

1 Moreira LF, Iwagaki H, Matsumo T et al. Submucosal lipoma synchronous with an early gastric cancer. J Clin Gastroenterol 1992; 14: $173-174$

2 Ono S, Fujishiro M, Goto $O$ et al. En bloc resection of cardia cancer and lipoma with endoscopic submucosal dissection. Dig Liver Dis 2009; 41: 237

3 Yamamoto T, Imakiire K, Hashiguchi S et al. A rare case of gastric lipoma with early gastric cancer. Intern Med 2004; 43: 1039-1041

Fig. 4 Macroscopic appearance of the surgically resected specimen showing a well-circumscribed, encapsulated tumor measuring $8.5 \times 5.5 \mathrm{~cm}$.
Bibliography

DOI http://dx.doi.org/

10.1055/s-0034-1377279

Endoscopy 2014; 46: E338-E339

(c) Georg Thieme Verlag KG

Stuttgart · New York

ISSN 0013-726X

\section{Corresponding author}

Tsutomu Namikawa, MD

Department of Surgery

Kochi Medical School

Kohasu, Oko-cho, Nankoku

Kochi 783-8505

Japan

Fax: +81-88-8802371

tsutomun@kochi-u.ac.jp 